\title{
ZNF139 promotes tumor metastasis by increasing migration and invasion in human gastric cancer cells
}

\author{
Y. LI*, B. B. TAN, Q. ZHAO, L. Q. FAN, D. WANG, Y. LIU \\ Department of General Surgery, the Fourth Affiliated Hospital, Hebei Medical University, 12 Jian-Kang St., 050011 Shijiazhuang, China \\ ${ }^{*}$ Correspondence: li_yong_hbth@126.com
}

Received April 4, 2013 / Accepted June 4, 2013

\begin{abstract}
Zinc finger protein 139(ZNF139), a member of zinc finger protein family, is a transcription factor. Our previous research showed ZNF139 was overexpressed in gastric cancer cells. The purpose of present study is to explore impact and mechanism of ZNF139 on metastasis by regulating invasive ability of gastric cancer cells.

Quantitative RT-PCR(QRT-PCR) and Western blot were applied for detection of ZNF139 expression in gastric cancer tissues, adjacent cancer tissues, metastatic lymph nodes, gastric cancer cell lines SGC7901 and BGC823 and gastric epithelial cell line GES-1; siRNA specific to ZNF139 was synthesized and then transfected into gastric cancer cell line BGC823; wound healing assay and Transwell assay were used to observe impact of ZNF139-siRNA after being transfected into BGC823 on its invasion and migration; changes in expression of invasion and migration-related genes MMP-2, MMP-9, ICAM-1 and TIMP1 were detected before and after transfection. Gelatin zymogrphy assay were applied to determine the MMP activities. Statistical analysis was based on the SPSS11.5 software.

Expression of ZNF139 in gastric adenocarcinoma tissues and cells was significantly higher than the expression in the adjacent cancer tissues, but lower than the expression in the metastatic lymph nodes; ZNF139 expression was present in gastric cancer cell lines, and the expression level was higher than that in normal gastric epithelial cells lines. ZNF139-siRNA significantly inhibited the invasion and migration activity of gastric cancer cell line BGC823. 48h after ZNF139-siRNA was transfected into gastric cancer cell line BGC823, expression and activity of invasion-related genes MMP-2, MMP-9, ICAM-1 mRNA and protein were significantly inhibited, while expressions of TIMP-1 mRNA and protein were significantly increased. At the same time, the gelatinase activities of MMP2 and MMP9 were decreased by ZNF139 interference.

ZNF139 was overexpressed in gastric cancer cells, and the expression was further enhanced in the metastasis process. Knocking down ZNF139 expression in gastric cancer cells could effectively reduce gastric cancer cell invasion and migration ability, and this process might play a role by regulating MMP-TIMP balance.
\end{abstract}

Key words: gastric cancer, zinc finger protein 139(ZNF139), migration, invasion, RNA interference technology

Gastric cancer is currently the second leading cancer killer in the world. High incidence of gastric cancer exists in east Asia, which is also the leading cause of death in malignant tumor in China. Gastric cancer has strong ability for proliferation, invasion and metastasis, which is one of the important factors that lead to poor prognosis. A variety of genes in tumor cells and their expression products play an important role in the invasion and metastasis process of gastric cancer [1-4]. It has been a hotspot to treat gastric cancer by inhibiting invasion and migration capability of gastric cancer cells, and the study has made some achievements [5-7]. But there has yet not been a breakthrough in the research, so it is of great significance to look for new genes that can regulate gastric cancer cell invasion ability for molecular targeted therapy of gastric cancer.

Zinc finger protein 139(ZNF139), a molecule identified from different differentiated gastric cancer tissues by two-dimensional difference gel electrophoresis and MODITOF-MS technology in our previous research, is a member of zinc finger protein family. Many members of zinc finger protein family play regulatory roles in a variety of genes at the transcriptional level, have important impacts on maintenance of normal life activities, and are closely related to tumorigenesis, development, metastasis and drug tolerance. Studies by Herman van Dekken et al [8] found that ZNF139 expression in adenocarcinoma at the esophagogastric junc- 
tion was increased, and was related to proliferation of tumor cells. Study also confirmed ZNF217 could promote tumor progression by accelerating ovarian cancer cell proliferation and invasion [9]; ZBP-89, a member of the Krüppel family, was related to distant metastasis and prognosis of renal cell carcinoma [10]. There were other studies that confirmed that the artificial transcription factor could be used for the detection of resistance genes and pathways, which might reverse the drug tolerance of tumors [11]. But there had been no report on relationship between ZNF139 and metastasis of gastric cancer cell in migration and invasion ability. In order to clarify the impact of ZNF139 on invasion and metastasis of gastric cancer cells, ZNF139 expression were detected in gastric cancer tissues, adjacent cancer tissues, metastatic lymph node tissues, gastric cancer cell lines and normal gastric epithelial cell line; and RNA interference technology was applied to the synthesis of ZNF139-siRNA, which was then transfected into gastric cancer cell line BGC823, to observe impact of inhibiting ZNF139 expression on gastric cancer cell invasion and migration activity, and to further explore the possible regulatory pathways.

\section{Materials and methods}

Patients and tissue specimens. 24 gastric adenocarcinoma tissue specimens were obtained from patients who were identified as aggressive gastric adenocarcinoma at the Fourth Affiliated Hospital of Hebei Medical University from April 2010 to April 2011. The 24 patients included 15 male and 9 female, with an average age of $(61.8 \pm 9.2)$ years. Hematoxylin and eosin (HE) staining on tumor tissues was done to determine histopathologic features. Samples were assigned a histological grade based on the World Health Organization (WHO) classification criteria. Gastric adenocarcinoma tissue specimens were divided into well differentiation and poor differentiation groups, each group contain 12 samples. The adjacent gastric mucosa tissues and metastasis lymph nodes were also collected from each case. Tissue specimens were preserved in $4 \%$ paraform or $-80^{\circ} \mathrm{C}$.

Cell culture. SGC7901 and BGC823 gastric cancer cell lines were obtained from Chinese Academy of Sciences Cell Bank and kept in our laboratory. All cell lines were cultured in Dulbecco's Modified Eagle Medium (DMEM) supplemented with $10 \%$ fetal calf serum (FCS) (Sigma Chemical, St. Louis, MO), $100 \mathrm{U} / \mathrm{ml}$ penicillin and $0.1 \mathrm{mg} / \mathrm{ml}$ streptomycin, $0.075 \%$ sodium bicarbonate and $200 \mu \mathrm{g} / \mathrm{ml}$ L-glutamine. All the cells were maintained in $5 \% \mathrm{CO}_{2}$ humidified atmosphere at $37^{\circ} \mathrm{C}$.

RNA interference. The cultured cells were grown to $50 \%$ confluence, and then transfected with ZNF139-specific siRNA (5'- ACCTCGGAAGATTCAGCAT-3') or non-specific NS-siRNA (5'-GACGAGTTGACTGCGATTG-3') using Lipofectamine $^{\mathrm{max}} 2000$ reagent (Invitrogen) according to the manufacturer's protocol. In brief, dilute siRNA oligomer in $50 \mu \mathrm{l}$ Opti-MEM I Reduced Serum Medium without serum and antibiotics, and mix gently. Mix Lipofectamine ${ }^{\mathrm{Tm}} 2000$ gently before use, then dilutes $1 \mu \mathrm{l}$ in $50 \mu \mathrm{l}$ Opti- MEM I Reduced Serum Medium. Mix gently and incubate for 5 minutes at room temperature, then combine the diluted oligomer with the diluted Lipofectamine ${ }^{\mathrm{mi}} 2000$. Mix gently and incubate for 20 minutes at room temperature, then add the mixture to each well containing cells and medium. Mix gently by rocking the plate back and forth. Incubated the cells at $37^{\circ} \mathrm{C}$ and changed medium 6 hours later.

Transwell assay. Cell migration activity was assayed in triplicate using a 24-well transwell (Corning Inc. USA) using polycarbonate Nucleopore filters with an 8 - $\mu \mathrm{m}$ pore size. Cells were transfected with ZNF139-siRNA or Control-siRNA for $24 \mathrm{~h}$, then were degested and resuspended. $5 \times 10^{4}$ cells were seeded in the upper chamber of each well. The lower chambers contained serum-free medium. The wells were incubated 16 $\mathrm{h}$ in cell incubator at $37^{\circ} \mathrm{C}$. Then non-migrated cells on the upper side of the membrane were removed by scraping, while migrated cells attached to the underside were fixed for $10 \mathrm{~min}$ in methanol and stained with crystal violet (Sigma). Cells were examined under a microscope and all cells in a specified area in the middle of the membrane were counted. Experiments were repeated three times.

Wound healing assay. Tumor cells seeded in 6-well plates were transfected with ZNF139-siRNA or Control-siRNA and grown to confluence. Then the plates were scratched within the confluent cell layer using the fine end of a sterile pipette tip. After 24,48 or $72 \mathrm{~h}$ incubation, cells migrated into the wounded region were counted under phase contrast microscopy. Experiments were repeated three times.

Total RNA isolation and quantitative RT-PCR. Total RNAs were isolated by one-step method using the Trizol reagent (Invitrogen) according to the manufacturer's instruction. The RNA samples were pretreated with RNase-free DNase, and $1 \mu \mathrm{g}$ RNA of each sample was reverse-transcribed to synthesis first strand cDNA using EasyScript First-Strand cDNA Synthesis Kit (TransGen Biotech, China). Then the cDNA templates were amplified by real-time quantitative PCR using Maxima SYBR Green/Fluorescein qPCR Master Mix (MBI, Canada) on an ABI-7300 PCR System (Applied Biosystems, USA). PCR reaction started with 1 cycle of $95^{\circ} \mathrm{C}$ for 10 minutes, followed by 40 cycles of three steps as $94^{\circ} \mathrm{C}$ for 30 seconds, $58^{\circ} \mathrm{C}$ for 30 seconds and $72^{\circ} \mathrm{C}$ for 30 seconds. The fluorescence data were collected at $72^{\circ} \mathrm{C}$ step. The mRNA expression of the target gene was normalized to the housekeeping glyceraldehyde-3-phosphate dehydrogenase (GAPDH) gene. The mRNA relative expression levels of target gene were represented as $2^{-\Delta \Delta C t}$ which were obtained by the software SDS v3.2 of ABI-7300 PCR system. PCR primers were designed using the Primer Express Software V3.0 (Applied Biosystems), and the sequences were as follows: ZNF139, 5'- CTTCCTGAGTTCTTGGTTTCG -3' (F) and 5'- CCTTTGACCCACTGGTTTATG -3' (R); MMP2, 5' - CAGGAGGAGAAGGCTGTGTT $-3^{\prime}(\mathrm{F})$ and 5'- AGGGTGCTGGCTGAGTAGAT - 3'(R); 
A

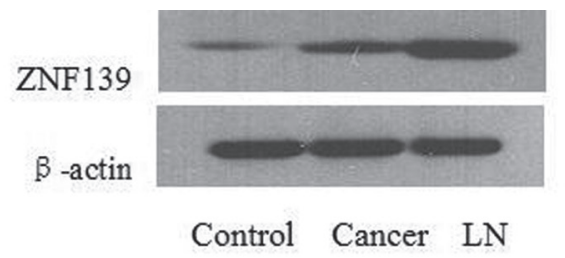

B
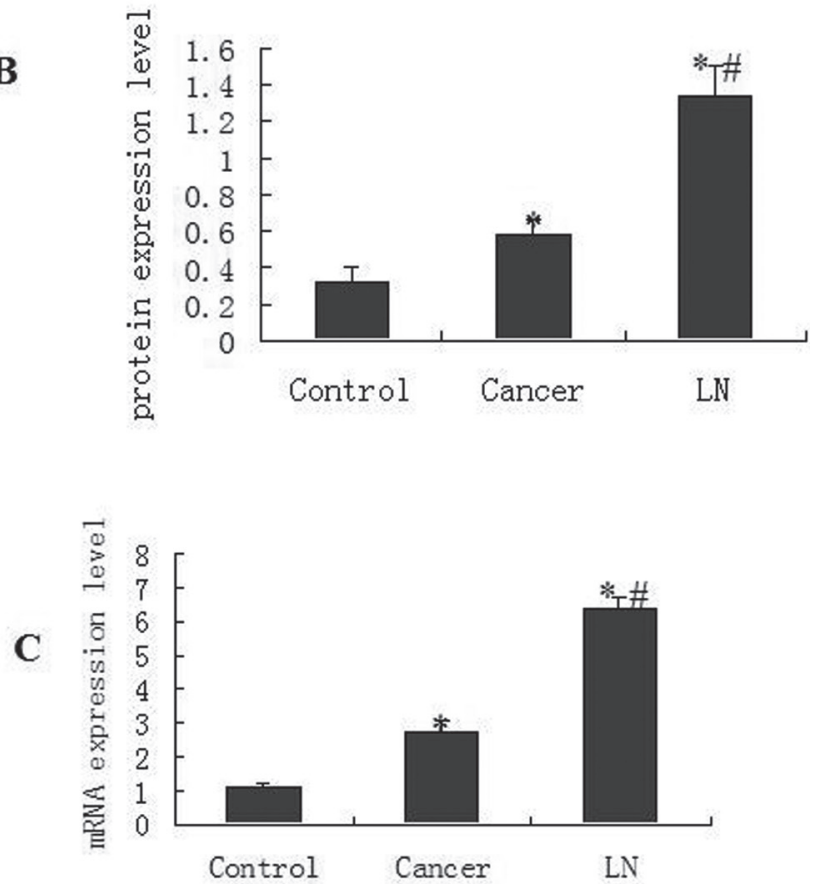

D

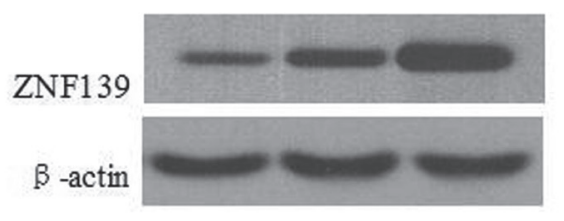

GES1 SGC7901 BGC823
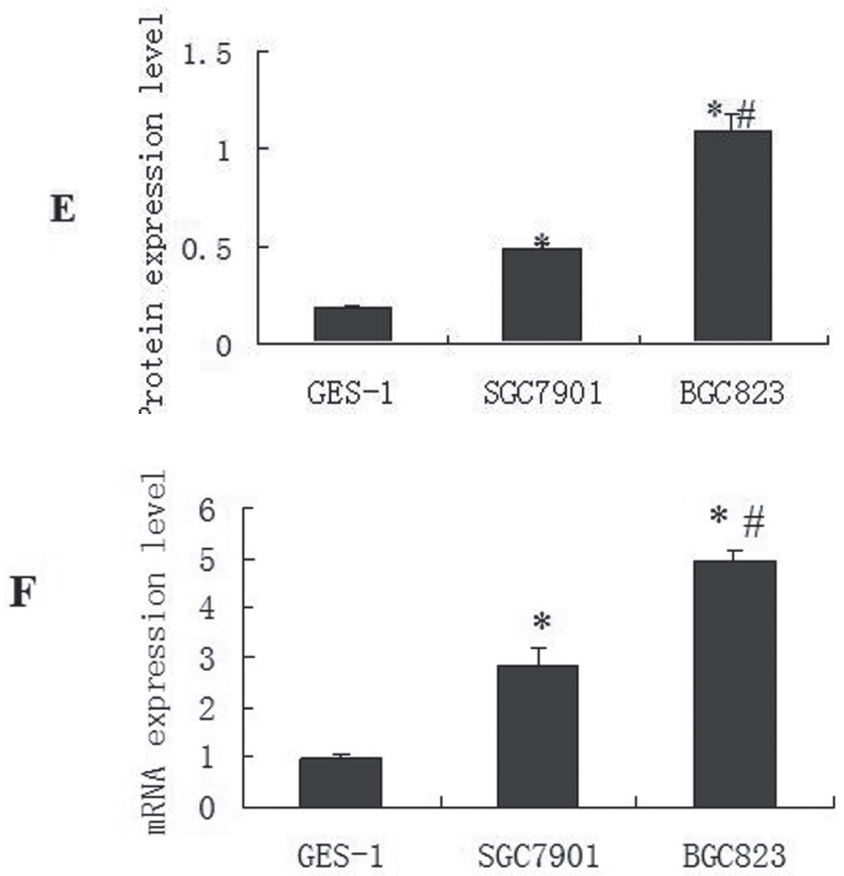

Fig1. ZNF139 expression level in gastric carcinomas and gastric cell lines Note: Clinical samples of gastric carcinomas, LNs and adjacent control normal tissues as well as GES1, SGC7901 and BGC823 cell lines were subjected to Western-blot (A and D) and quantitative RT-PCR (C and F) assays to determine the expression of ZNF139. The relative protein expression levels were shown in $B$ and $E$. Values were shown as mean $\pm S D$, for tissue samples $n=6$, cell samples $n=4$ in each group. ${ }^{\star} P<0.01$ versus control group; $\# P<$ 0.01 versus cancer or SGC7901 group.

MMP9, 5' - AGAACCAATCTCACCGACAGG -3' (F) and 5'- CGACTCTCCACGCATCTCT - 3'(R); TIMP1, 5'- ACTTCCACAGGTCCCACAAC - 3' (F) and 5'GCATTCCTCACAGCCAACAG - 3'(R); ICAM1, 5'- GGAAGGTGTATGAACTGAGCAAT -3' (F) and 5'- TGGCAGCGTAGGGTAAGGT - 3'(R); GAPDH, 5'- GACCCCTTCATTGACCTCAAC-3' (F) and 5'- CGCTCCTGGAAGATGGTGAT- 3'(R).

Western-blot assay. Tissue and cell samples were lysated with lysis buffer (1\% Triton X-100, $150 \mathrm{mM} \mathrm{NaCl}, 10 \mathrm{mM}$ Tris-HCl, pH 7.4, 1 mM EDTA, 1 mM EGTA, pH 8.0, 0.2 mM $\mathrm{Na} 3 \mathrm{VO} 4,0.2 \mathrm{mM}$ phenylmethylsulfonyl fluoride, and $0.5 \%$ NP-40). Protein concentration was determined by Bradford assay (Bio-Rad Laboratories). Equal amounts of protein for each sample were separated by $10 \%$ polyacrylamide SDS gels (SDS-PAGE) and electrotransferred to a polyvinylidene fluoride (PVDF) membrane (Amersham Pharmacia Biotech). Membranes were blocked with 5\% BSA for $2 \mathrm{~h}$ at room temper- ature, and incubated with the 1:300 diluted primary antibody of ZNF139,MMP9, MMP2, TIMP1, ICAM1 or GAPDH (all from Santa Cruz, USA) overnight at $4^{\circ} \mathrm{C}$. Membranes were incubated for $2 \mathrm{~h}$ in a horseradish peroxidase-conjugated secondary antibody (1:10,000 diluted), target bands were detected with the enhanced chemiluminescence (ECL) detection system (Santa Cruz, USA) according to the manufacturer's instructions. GAPDH was used as the loading control. The experiments were replicated three times.

Gelatin zymogrphy assay. Each $30 \mu$ of culture medium from cells transfected with ZNF139-siRNA or control NSsiRNA was mixed with SDS loading buffer and incubated for $10 \mathrm{~min}$ at room temperature, then were separated by $10 \%$ SDSPAGE gel which was prepared with the addition of $10 \mathrm{mg} / \mathrm{ml}$ gelatin. After electrophoresis, the gel was incubated 3 times for $30 \mathrm{~min}$ in washing buffer $(50 \mathrm{mM}$ Tris/ $\mathrm{HCl} \mathrm{pH} \mathrm{7.5,5} \mathrm{mM}$ $\mathrm{CaCl} 2$ and $2.5 \%$ triton $\mathrm{X}-100)$ to get rid of the SDS from the gel. Then the gel was incubated in the reaction buffer $(50 \mathrm{mM}$ 
Tris/ $\mathrm{HCl} \mathrm{pH} \mathrm{7.5,} 5 \mathrm{mM} \mathrm{CaCl}_{2}$ ) overnight at $37^{\circ} \mathrm{C}$, and the gel was stained with Coomassie brilliant blue.

Data statistics.Data are presented as means \pm S.E. Statistical analyses were carried out with the SPSS 11.5 statistical software package. Our primary statistical test was the one-way ANOVA. $P<0.05$ was considered statistically significant.

\section{Results}

ZNF139 expression in gastric cancer tissues and cell lines. We first applied quantitative RT-PCR (QRT-PCR) and Western blot to detection of ZNF139 expression in clinical samples of gastric cancer, adjacent cancer tissues, metastasis lymph nodes. Similarly, QRT-PCR and Western blot were used to detect ZNF139 expression of the gastric cancer cell lines. Results showed that, compared with adjacent cancer tissues of cancer, expression of ZNF139 in gastric cancer was up-regulated, whereas expression of ZNF139 in metastasis lymph nodes was stronger than in primary cancer tissues $(P<0.05)$. Also expression of ZNF139 was stronger in the

A

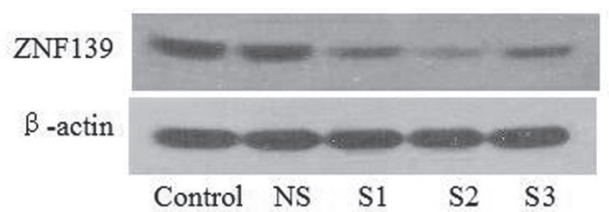

B

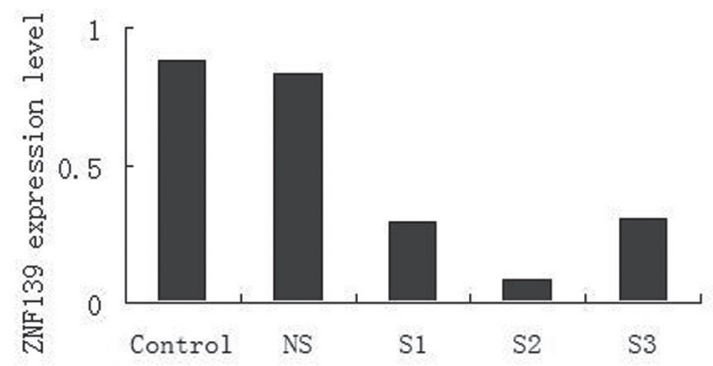

C

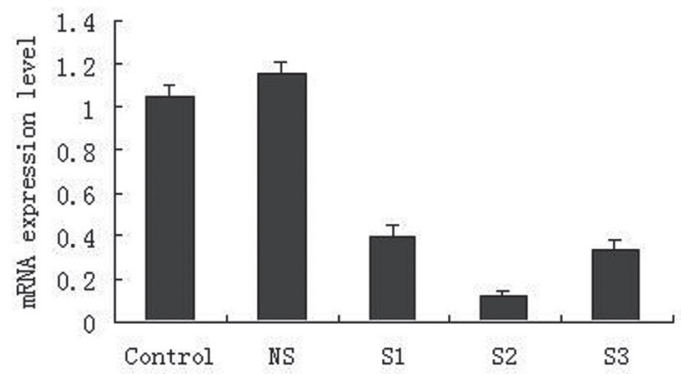

human gastric cancer cell lines (SGC7901, BGC823) than in gastric epithelial cell line GSE-1 $(P<0.05)$, and expression of ZNF139 was stronger in BGC823 than in SGC7901; results from QRT-PCR and Western blot test were consistent. These results showed that ZNF139 expression in gastric cancer cells was significantly up-regulated, suggesting that ZNF139 might play a role in invasion and metastasis of gastric cancer.

Impact of ZNF139-siRNA on ZNF139 of BGC823 cells. In order to clarify the impact of ZNF139 on gastric carcinogenesis, we designed 3 pairs of ZNF139-siRNAs with different sequence, and transfected them into gastric cancer cell line BGC823, in which overexpressed ZNF139 was detected, with transfected control-siRNA cells as negative control. QRT-PCR and Western-blot results showed that ZNF139 expression did not change after being transfected with control-siRNA; and after being transfected 3 pairs of Vav3-siRNA cells, mRNA and protein of ZNF139 in BGC823 cells were inhibited significantly, in which, ZNF139 in BGC823 cells transfected with ZNF139-siRNA-2 dropped greatly at approximately 90\% (Fig.2 $A, B)$, indicating that ZNF139-siRNA used in this study could

D

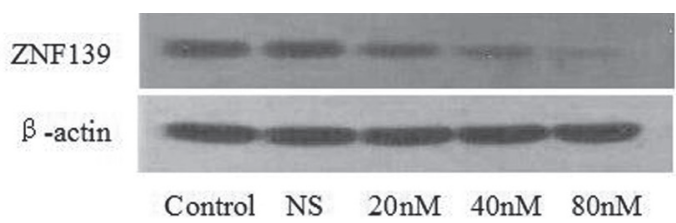

$\mathbf{E}$
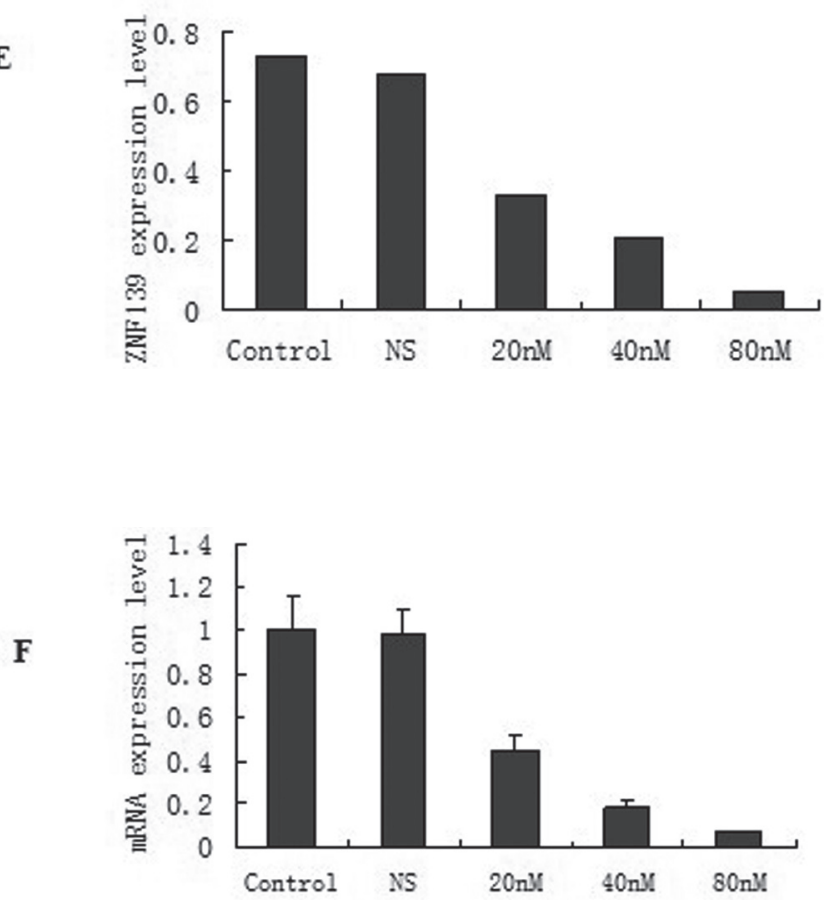

Fig 2. ZNF139-siRNA down-regulated ZNF139 expression in BGC823 cells Note: Cells were transfected with different sequence of ZNF139-siRNA (S1, S2, S3) or control NS-siRNA (A, B and C), alternatively, cells were transfected with different amount of the sequence of ZNF139-siRNA-2 for 48 hours (D, E and F), the expression of ZNF139 were identified by Western-blot (A, B and D, E) as well as quantitative RT-PCR (C and F). The expression levels were represented as columns in B and E. 
effectively inhibit the transcription and translation of endogenous ZNF139. $20 \mathrm{nM}, 40 \mathrm{nM}$ and $80 \mathrm{nM}$ of ZNF139-siRNA-2 were further transfected into BGC823 cells, and QRT-PCR and Western-blot results showed ZNF139 expression in cells had a concentration-dependent decrease, in which after $80 \mathrm{nM}$ of ZNF139-siRNA-2 was transfected, ZNF139 expression in cells was decreased by over $95 \%$ (Fig.2 C and D).

Impact of ZNF139-siRNA on invasion and migration of BGC823 cells. After $20 \mathrm{nM}, 40 \mathrm{nM}$ and $80 \mathrm{nM}$ of ZNF139siRNA-2 were transfected into BGC823 cells, cell migration activity changes were observed with wound healing assay, and changes in cell invasion ability was detected in Transwell assay. As was shown in Fig. 3 A and B, compared with the negative control group transfected with control-siRNA, migration inhibition rate and invasion inhibition rate had a dose-dependent increase in BGC823 cells transfected with ZNF139-siRNA $(P<0.05)$, suggesting that inhibition of ZNF139 could inhibit invasion and migration of BGC823 cells. Compared with liposome control group, migration inhibitory rate and invasion inhibition rate was not statistically significant $(P>0.05)$ in negative control group transfected with control-siRNA.

Impact of ZNF139-siRNA on expression of MMP-2, MMP-9, ICAM-1, TIMP-1 in BGC823 cells. Subsequently, real-time quantitative RT-PCR and Western blot were applied to detection of invasion- and migration-related gene expressions and the results showed that, compared with the negative control group transfected with control-siRNA, after $80 \mathrm{nM}$ of ZNF139-siRNA-2 was transfected into BGC823 cells, expressions of TIMP-1 was significantly up-regulated, while expressions of MMP-2, MMP-9, ICAM-1 were significantly decreased (Fig.4). The results of gelatin zymography assay show that the gelatinolytic activities of MMP2 and MMP9 were also inhibited by ZNF139-siRNA transfection. These results suggested that ZNF139 was able to promote gastric cancer cell migration and invasion, and inhibition of ZNF139 expression could inhibit gastric cancer cell migration and invasion.

\section{Discussion}

Gastric cancer is the most common digestive carcinoma in China, and the mortality rate is among the highest in a variety of malignant tumors, seriously threatening the life and health of the people $[12,13]$. Because of gastric occult onset and strong migration and invasion ability of tumor cells, frequently there has been a regional lymph node metastasis or distant metastasis before treatment, leading to poor comprehensive treatment effects by surgery, radiotherapy and chemotherapy. Detection rate of Chinese patients with early gastric cancer was less than $10 \%[14,15]$; most patients have been found to have advanced cancers, and $50 \%$ to $80 \%$ of patients had lymph node metastasis or other organ invasion and metastasis when undergoing surgery. The 5-year survival rate of advanced gastric cancer has been fluctuating at around $40 \%-50 \%[16,17]$. If appropriate measures could be taken to reduce the ability of tumor cell invasion and metastasis, it will be of great significance in
A

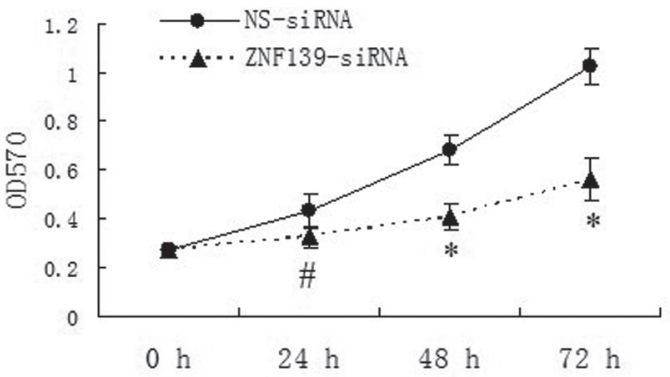

B

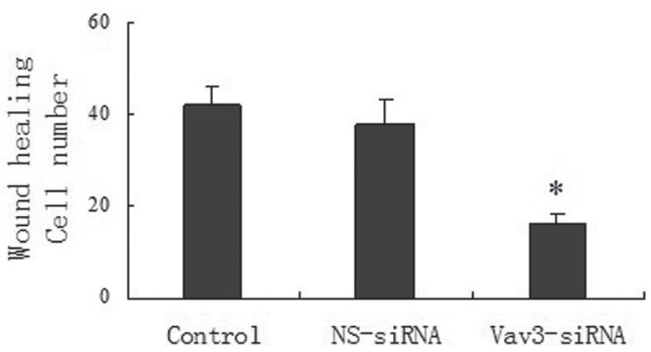

C

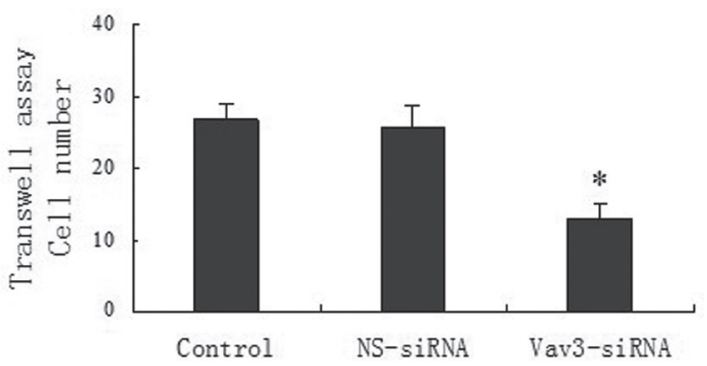

Fig 3. Effects of ZNF139 on proliferation, migration, invasion of BGC823 cells

Note: Cells were transfected with ZNF139-siRNA or control NS-siRNA, then were subjected to (A) wound healing assays to detect cell migrating activity, (B) transwell assays to detect cell invasion activity. ${ }^{\star} \mathrm{P}<0.01$ and $\# \mathbf{P}<0.05$ versus control group.

delaying the gastric carcinogenesis and in improvement of treatment and prognosis. It has been a hot research to study characteristics of proliferation, differentiation, invasion and metastasis of different differentiated gastric cancer cells and to study a variety of genes as well as function and interplay of their expression products in it; but so far there has yet not been a breakthrough in the research, nor key genes that impacted gastric cancer invasion and metastasis were found.

In previous work of this study, MALDF-TOF-MS was used to successfully screen and identify a variety of differentiation-associated proteins from different differentiated gastric cancer cell lines, including ZNF139. Studies by Herman van Dekken et al [8] also found increased expression of ZNF139 in adenocarcinoma at the esophagogastric junction. ZNF139, a member of zinc finger protein family, contains six $\mathrm{C}_{2} \mathrm{H}_{2}$ zinc finger domains, a SCAN and KRAB (krüppel-associated box) 
A
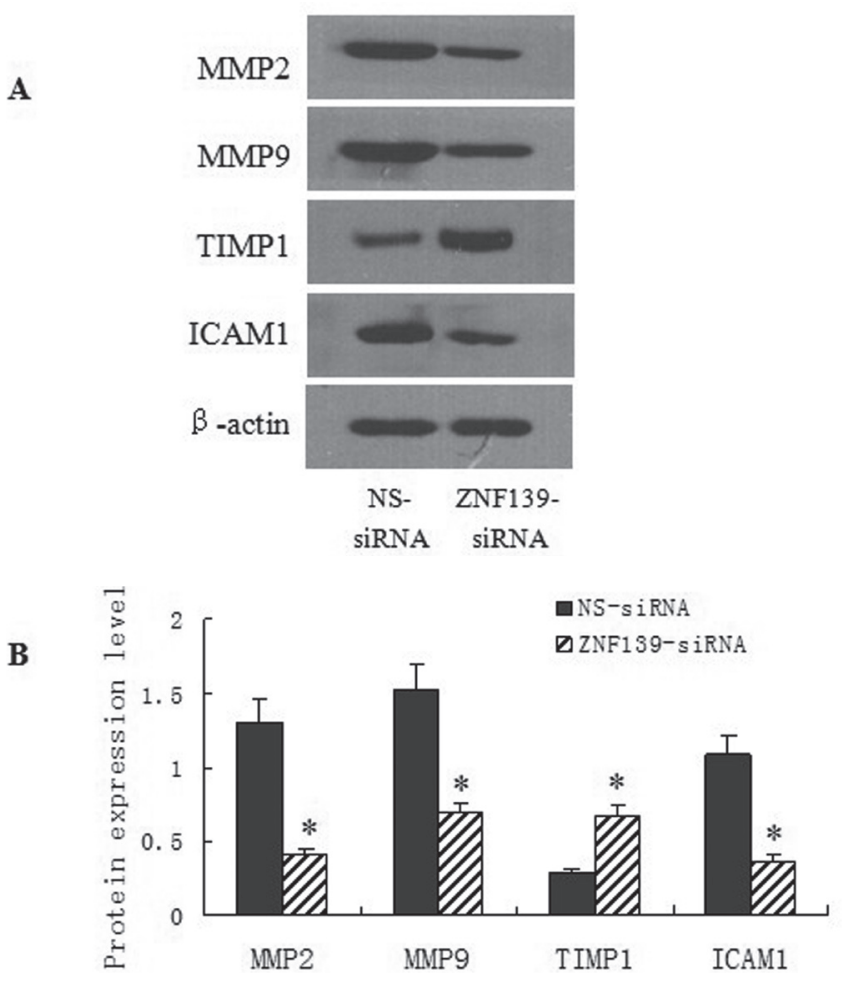

C

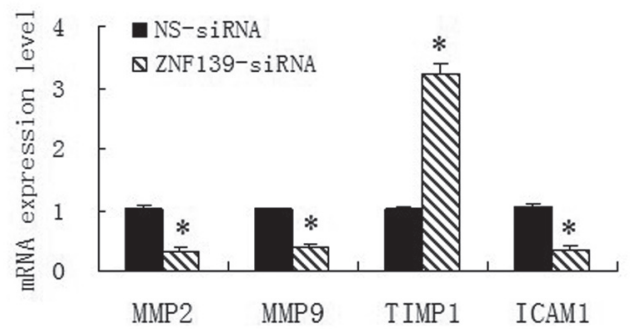

D

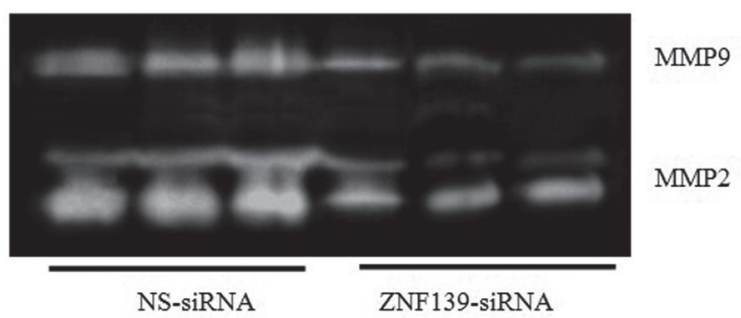

Fig 4. Effects of ZNF139 on migration, invasion related genes in BGC823 cells

Note: Cells were transfected with ZNF139-siRNA or control NS-siRNA, then were subjected to Western-blot assays (A) or quantitative RT-PCR (C) assays to detect the protein or mRNA expression levels of MMP-2, MMP-9, ICAM-1 and TIMP-1. The protein expression levels were represented as columns in B. (D) Gelatin zymogrphy was employed to assay the MMP2 and MMP9 activity. ${ }^{*} \mathbf{P}<0.01$ versus NS-siRNA control group.

domains. Zinc finger is zinc finger DNA-binding domain, while SCAN and KRAB mediate protein-protein interactions between the cofactors, which perform the transcriptional regu- lation of downstream gene expression by binding themselves to a target gene promoter region [18]. Although there has been no report on relationship between ZNF139 and invasion and metastasis of gastric cancer, but there have been many researches on relationship between zinc finger protein family and tumor invasion and metastasis $[19,20]$. Therefore, we studied ZNF139 expression in gastric cancer and its relationship with gastric cancer invasion and metastasis.

In the experiment, ZNF139 expression in gastric cancer tissues and cells was significantly higher than the expression in adjacent cancer tissues, and there was further increased expression in metastatic lymph nodes, indicating ZNF139 might be used as the gastric tumor markers, and could predict metastasis of gastric cancer, which was worth further study. Because tumor invasion and metastasis is an extremely complex pathological process with multiple steps and multiple factors involved, adhesion between cancer cells, adhesion between cancer cells and the extracellular matrix, adhesion between cancer cells and the normal cells, the degradation of the extracellular matrix, and cancer cell movement migratory ability play an important role, which are the key factors determining whether the malignant tumor will have invasion and metastasis $[21,22]$. Our study confirmed ZNF139 expression was closely related to gastric cancer cell migration activity; after silencing ZNF139 expression specific small interfering RNA could inhibit migration activity of BGC823 cell.

Degradation of extracellular matrix (ECM) and destruction of the integrity of the basement membrane are a prerequisite for invasion and metastasis ability of tumor cells. The degradation of extracellular matrix could be finished by proteolytic enzymes like matrix metalloproteinases (MMPs); activity of MMPs can be inhibited by metal matrix protease inhibitors (TIMPs), its natural inhibitor; MMPs-TIMPs balance is the determinant of ECM internal environment and integrity maintenance [23-25]. Overexpression of 72KD of MMP-2 and $92 \mathrm{KD}$ of MMP-9 in Type IV collagenase from MMP family is closely related to a variety of malignant tumor invasion and metastasis [26, 27]. TIMP-1 from TIMPs has a strong affinity with MMP-9, and plays a direct or indirect inhibition role in other members from MMPs family [28]. ICAM-1, a glycoprotein, can promote migration and invasion of tumor cells, and plays an important role in tumor invasion and migration $[29,30]$. Our findings showed that after ZNF139 expression, expressions of MMP-2, MMP-9 and ICAM-1 were decreased, while TIMP-1 expression was increased, suggesting that ZNF139 gene was closely related to invasion and metastasis of gastric cancer and its regulatory mechanism might be caused by adjusting MMP-TIMP balance and ICAM-1.

\section{Conclusion}

Present study found that overexperssion of ZNF139 was ralted to invasion and migration of gastric cancer cells. 
After siRNA technology was applied to silencing ZNF139, expressions of MMP-2, MMP-9 and ICAM-1 were decreased, while TIMP-1 expression was increased, thereby inhibiting invasion and migration ability of gastric cancer cells. ZNF139 gene may be a new molecule involved in the regulation of gastric cancer cell invasion activity, regulating the gastric cancer cell invasion activity by affecting MMP/ TIMP balance. Therefore, ZNF139 may play an important role in the regulation of gastric cancer invasion and migration process, and become predicted genes in gastric cancer metastasis as well as the target gene in biological treatment of gastric cancer. The specific molecular mechanisms should be further studied.

Acknowledgments: This work was supported by grants as follows: The National Natural Science Foundation of China (No.81072033 to Yong Li); The Provincial Natural Science Foundation of Hebei Province (No. C2010000619 to Yong Li); Extra Characteristic Foundation of Colleges and Universities in Hebei Province (NO. [2005]52, to Yong $\mathrm{Li}$ )

\section{References}

[1] LEE JH, CHOI MG, MIN BH, NOH JH, SOHN TS et al: Predictive factors for lymph node metastasis in patients with poorly differentiated early gastric cancer. Br J Surg 2012; 99; 1688-1692. http://dx.doi.org/10.1002/bjs.8934

[2] CHOI JH, SHIN NR, MOON HJ, KWON CH, KIM GH et al: Identification of S100A8 and S100A9 as negative regulators for lymph node metastasis of gastric adenocarcinoma. Histol Histopathol 2012; 27; 1439-1448.

[3] ZHU ZL, YAN BY, ZHANG Y, YANG YH, WANG ZM et al: PINCH expression and its clinicopathological significance in gastric adenocarcinoma. Dis Markers 2012; 33; 171-178. http://dx.doi.org/10.1155/2012/895713

[4] CHEN P, ZHAO D, SUN Y, HUANG L, ZHANG S et al: Protein inhibitor of activated STAT-1 is downregulated in gastric cancer tissue and involved in cell metastasis. Oncol Rep 2012; $28 ; 2149-2155$.

[5] MA PF, CHEN JQ, WANG Z, LIU JL, LI BP: Function of chloride intracellular channel 1 in gastric cancer cells. World J Gastroenterol 2012; 18; 3070-3080. http://dx.doi.org/10.3748/ wjg.v18.i24.3070

[6] KUNDU J, WAHAB SM, KUNDU JK, CHOI YL, ERKIN $\mathrm{OC}$ et al: Tob1 induces apoptosis and inhibits proliferation, migration and invasion of gastric cancer cells by activating Smad4 and inhibiting $\beta$-catenin signaling. Int J Oncol 2012; $41 ; 839-848$.

[7] ZHANG J, ZHU JS, ZHOU Z, CHEN WX, CHEN NW: Inhibitory effects of ethyl pyruvate administration on human gastric cancer growth via regulation of the HMGB1-RAGE and Akt pathways in vitro and in vivo. Oncol Rep 2012; 27; 1511-1519.

[8] VAN DEKKEN H, TILANUS HW, HOP WC, DINJENS WN, WINK JC et al: Array comparative genomic hybridization, expression array, and protein analysis of critical regions on chromosome arms 1q, 7q, and 8p in adenocarcinomas of the gastroesophageal junction. Cancer Genet Cytogenet 2009; 189; 37-42. http://dx.doi.org/10.1016/j.cancergencyto.2008. $\underline{08.018}$

[9] SUN G, ZHOU J, YIN A, DING Y, ZHONG M: Silencing of ZNF217 gene influences the biological behavior of a human ovarian cancer cell line. Int J Oncol 2008; 32; 1065-1071.

[10] CAI MY, LUO RZ, LI YH, DONG P, ZHANG ZL et al: Highexpression of ZBP-89 correlates with distal metastasis and poor prognosis of patients in clear cell renal cell carcinoma. Biochem Biophys Res Commun 2012; 426; 636-642. http:// dx.doi.org/10.1016/j.bbrc.2012.08.146

[11] BLANCAFORT P, TSCHAN MP, BERGQUIST S, GUTHY D, BRACHAT A et al: Modulation of drug resistance by artificial transcription factors. Mol Cancer Ther 2008; 7; 688-697. http://dx.doi.org/10.1158/1535-7163.MCT-07-0381

[12] ZHENG KC, AOKI K, LI XQ, LIN SG, WU BS et al: Serum Pepsinogens, Gastrin-17 and Helicobacter pylori Antibody in the Residents of Two Cities in China with Distinct Mortality Rates of Gastric Cancer. Tohoku J Exp Med 2012; 228; 289-294. http://dx.doi.org/10.1620/tjem.228.289

[13] WANG HM, HUANG CM, ZHENG CH, LI P, XIE JW et al: Tumor size as a prognostic factor in patients with advanced gastric cancer in the lower third of the stomach. World J Gastroenterol 2012; 18; 5470-5475. http://dx.doi.org/10.3748/wjg. v18.i38.5470

[14] HUANG B, WANG Z, XING C, SUN Z, ZHAO B et al: Longterm survival results and prognostic factors of early gastric cancer. Exp Ther Med 2011; 2; 1059-1064.

[15] LIAN J, CHEN S, ZHANG Y, QIU F: A meta-analysis of endoscopic submucosal dissection and EMR for early gastric cancer. Gastrointest Endosc 2012; 76; 763-770. http://dx.doi. org/10.1016/j.gie.2012.06.014

[16] DING YB, XIA TS, WU JD, CHEN GY, WANG S et al: Surgical outcomes for gastric cancer of a single institute in southeast China. Am J Surg 2012; 203; 217-221. http://dx.doi. org/10.1016/j.amjsurg.2010.10.022

[17] LIU E, ZHONG M, XU F, LIU W, HUANG J et al: Impact of lymphatic vessel invasion on survival in curative resected gastric cancer. J Gastrointest Surg 2011; 15; 1526-1531. http:// dx.doi.org/10.1007/s11605-011-1600-0

[18] TOMMERUP N, VISSING H: Isolation and fine mapping of 16 novel human zinc finger-encoding cDNAs identify putative candidate genes for developmental and malignant disorders. Genomics 1995; 27; 259-264. http://dx.doi.org/10.1006/ geno.1995.1040

[19] TSAI SJ, HWANG JM, HSIEH SC, YING TH, HSIEH YH: Overexpression of myeloid zinc finger 1 suppresses matrix metalloproteinase- 2 expression and reduces invasiveness of SiHa human cervical cancer cells. Biochem Biophys Res Commun 2012; 425; 462-467. http://dx.doi.org/10.1016/ j.bbrc.2012.07.125

[20] RAHMAN MT, NAKAYAMA K, RAHMAN M, KATAGIRI $\mathrm{H}$, KATAGIRI A et al: Gene amplification of ZNF217 located at chr20q13.2 is associated with lymph node metastasis in ovarian clear cell carcinoma. Anticancer Res 2012; 32; 3091-3095. 
[21] CAO DX, LI ZJ, JIANG XO, LUM YL, KHIN E et al: Osteopontin as potential biomarker and therapeutic target in gastric and liver cancers. World J Gastroenterol 2012; 18; 3923-3930. http://dx.doi.org/10.3748/wjg.v18.i30.3923

[22] LI Z, MIAO Z, JIN G, LI X, LI H et al: $\beta$ ig-h3 supports gastric cancer cell adhesion, migration and proliferation in peritoneal carcinomatosis. Mol Med Report 2012; 6; 558-564.

[23] KAMOSHIDA G, MATSUDA A, SEKINE W, MIZUNO H, OKU T et al: Monocyte differentiation induced by co-culture with tumor cells involves RGD-dependent cell adhesion to extracellular matrix. Cancer Lett 2012; 315; 145-152. http:// dx.doi.org/10.1016/j.canlet.2011.09.029

[24] LUKASZEWICZ-ZAJAC M, MROCZKO B, SZMITKOWSKI $\mathrm{M}$ : Gastric cancer - The role of matrix metalloproteinases in tumor progression. Clin Chim Acta 2011; 412; 1725-1730. http://dx.doi.org/10.1016/j.cca.2011.06.003

[25] OSINSKY S, BUBNOVSKAYA L, GANUSEVICH I, KOVELSKAYA A, GUMENYUK L et al: Hypoxia, tumour-associated macrophages, microvessel density, VEGF and matrix metalloproteinases in human gastric cancer: interaction and impact on survival. Clin Transl Oncol 2011; 13; 133-138. http://dx.doi.org/10.1007/s12094-011-0630-0

[26] JEZIERSKA A, MOTYL T: Matrix metalloproteinase-2 involvement in breast cancer progression: a mini-review. Med Sci Monit 2009; 15; RA32-40.

[27] VELINOV N, POPTODOROV G, GABROVSKI N, GABROVSKI S: The role of matrixmetalloproteinases in the tumor growth and metastasis. Khirurgiia (Sofiia) 2010; 44-49.

[28] ZHANG M, ZHU GY, GAO HY, ZHAO SP, XUE Y: Expression of tissue levels of matrix metalloproteinases and tissue inhibitors of metalloproteinases in gastric adenocarcinoma. J Surg Oncol 2011; 103; 243-247. http://dx.doi.org/10.1002/jso.21824

[29] JUNG WC, JANG YJ, KIM JH, PARK SS, PARK SH et al: Expression of intercellular adhesion molecule-1 and e-selectin in gastric cancer and their clinical significance. J Gastric Cancer 2012; 12; 140-148. http://dx.doi.org/10.5230/jgc.2012.12.3.140

[30] SUN DZ, XU L, WEI PK, LIU L, HE J: Syndrome differentiation in traditional Chinese medicine and E-cadherin/ICAM-1 gene protein expression in gastric carcinoma. World J Gastroenterol 2007; 13; 4321-4327. 